\title{
UMA NOVA ABORDAGEM BRANCH AND CUT APLICADA AO PROBLEMA DE PLANEJAMENTO DA EXPANSÃO DE REDES DE TRANSMISSÃO DE GRANDE PORTE
}

\author{
Aldir Silva Sousa* \\ aldirss@usp.br
}

\author{
Eduardo Nobuhiro Asada* \\ easada@usp.br
}

\author{
*Universidade de São Paulo \\ Escola de Engenharia de São Carlos \\ Departamento de Engenharia Elétrica \\ São Carlos-SP 13566-590
}

\begin{abstract}
A New Branch and Cut Approach Applied to the LongTerm Transmission Expansion Planning Problem

In this paper we propose a branch and cut algorithm with new inequality constraints applied to the long-term transmission network expansion problem. All the proposed inequalities in this research work are valid for both linear and nonlinear models of the problem. The computational results have demonstrated the effectiveness of the proposed method when applied to Brazilian and Colombian systems.
\end{abstract}

KEYWORDS: Transmission expansion planning, cutset inequalities, branch and cut method.

\section{RESUMO}

Neste artigo propomos um algoritmo branch and cut com novas inequações específicas ao problema de planejamento da expansão de redes de transmissão de energia elétrica. Todas as inequações propostas neste trabalho são válidas tanto para os modelos lineares como para os modelos não lineares do problema. Os testes computacionais têm mostrado a eficiência do método proposto neste trabalho quando aplicado a subsistemas reais brasileiros e ao sistema colombiano.

Artigo submetido em 24/08/2010 (Id.: 01189)

Revisado em 28/08/2010, 11/02/2011, 11/05/2011, 13/05/2011

Aceito sob recomendação do Editor Associado Prof. Julio Cesar Stacchini Souza
PALAVRAS-CHAVE: Planejamento da Expansão da Transmissão, Método branch and cut.

\section{INTRODUÇÃO}

O problema de planejamento da expansão de sistemas de transmissão de energia elétrica (PEST) tem como objetivo determinar o custo de investimento mínimo para a expansão do sistema de transmissão de energia visando atender o aumento da demanda no horizonte futuro. O PEST é executado depois do estudo de previsão da geração e da demanda de energia elétrica. Após determinado em quanto e onde o sistema terá aumento de geração e demanda, surge o problema de determinar o menor custo de adição de equipamentos (linhas de transmissão e/ou transformadores) de tal forma que a demanda seja atendida com segurança.

Com a reorganização da indústria de energia elétrica, novos desafios têm sido observados. Um desses desafios é considerar a perspectiva temporal do investimento no contexto de problema de otimização. Quando não se considera o tempo, a solução do problema apenas determina quais equipamentos devem ser adicionados ao sistema. Assim, pode-se afirmar que há somente um plano de planejamento (planejamento estático). Quando há uma perspectiva temporal, a solução deve determinar também o momento em que os equipamentos devem ser instalados na rede. Dessa forma, o período de planejamento deve ser dividido em sub-períodos, sendo necessário determinar quais equipamentos devem ser adicio- 
nados em cada um deles. A abordagem em que se consideram os sub-períodos é chamada de planejamento multiestágio (Garver, 1970; Romero et al., 2007). Os artigos (Garcia et al., 1997; Romero et al., 2003; Escobar et al., 2004) são exemplos de trabalhos que consideram o planejamento multiestágio.

As dificuldades na solução deste problema são devidas a sua natureza combinatória, às restrições de integralidade de algumas variáveis e à não-linearidade do problema. As restrições essenciais são derivadas das duas leis de Kirchhoff. A lei das tensões de Kirchhoff (LTK) adiciona não-linearidade ao problema. Para contornar a não-linearidade, uma das primeiras propostas bem-sucedidas para solução do PEST foi apresentado em (Garver, 1970), em que foi proposto o modelo de transportes para modelar o problema.

As metodologias sugeridas para solução do PEST podem ser divididas em dois grandes grupos: o grupo dos métodos exatos e os métodos aproximados. Dentre os métodos exatos, o algoritmo branch and bound (B\&B) e os métodos de decomposição têm sido os mais empregados. Os artigos (Romero and Monticelli, 1994; Asada et al., 2006; Choi et al., 2005; Haffner et al., 2000; Haffner et al., 2001; Levi and Calovic, 1991) são exemplos de contribuições para resolução do PEST por meio de métodos exatos.

Dentre os métodos aproximados, os mais empregados são os métodos heurísticos construtivos e as metaheurísticas. Os métodos heurísticos construtivos, quando lidam com sistemas de grande porte, encontram soluções em pouco tempo computacional, porém geralmente de baixa qualidade. Quando se consideram sistemas de grande porte, as metaheurísticas têm apresentado os melhores resultados

com relação a qualidade de solução. Os trabalhos (Garver, 1970; Monticelli et al., 1982; Pereira and Pinto, 1985; Villasana et al., 1985; Romero et al., 1996; Da Silva et al., 2000; Binato et al., 2001; Sousa and Asada, 2008) são contribuições para a solução do PEST utilizando métodos aproximados. Quando se aborda o PEST com suas características não determinísticas, sistemas fuzzy e programação multiobjetivo (Buygi et al., 2004; Chung et al., 2003; Choi et al., 2005) têm sido bastante aplicados.

Neste trabalho propomos inequações e conjuntos de cortes válidos para os modelos do PEST e uma nova estrutura branch and cut (B\&C) para solução de sistemas de grande porte em um ambiente de planejamento centralizado. Testes foram realizados considerando o modelo híbrido, proposto em (Villasana et al., 1985) e detalhado na Seção 2, para sistemas reais (sistemas brasileiros e colombianos).

Este trabalho está organizado da seguinte forma. Na Seção 2 , são apresentados alguns dos principais modelos que re- presentam o PEST; na Seção 3, é apresentado sucintamente a definição do método branch and cut e são apresentadas as inequações válidas para o modelo híbrido do PEST; na Seção 4, é apresentado o algoritmo usado para construir caminhos no sistema e deles construir as cutset inequalities; na Seção 5 , são apresentados os resultados obtidos com o método proposto; na Seção 6 é feita uma análise sucinta dos resultados obtidos e na Seção 7, são apresentadas as principais conclusões deste trabalho.

\section{MODELOS MATEMÁTICOS}

Dentre os modelos encontrados na literatura para representar o problema de planejamento da expansão, o modelo cc é considerado o mais apropriado segundo (Monticelli et al., 1982), sendo modelado matematicamente como segue:

$$
\min v=\sum_{(i, j) \in \Omega} c_{i j} n_{i j}
$$

s.a.

$$
\begin{gathered}
S f+g=d \\
f_{i j}-\gamma_{i j}\left(n_{i j}^{0}+n_{i j}\right)\left(\theta_{i}-\theta_{j}\right)=0 \forall(i, j) \in \Omega \\
\left|f_{i j}\right| \leq\left(n_{i j}^{0}+n_{i j}\right) \bar{f}_{i j} \forall(i, j) \in \Omega \\
0 \leq g \leq \bar{g} \\
0 \leq n \leq \bar{n}_{i j} \\
n_{i j} \text { inteiro; } f_{i j} \text { irrestrito } \forall(i, j) \in \Omega ; \\
\theta_{j} \text { irrestrito } \forall j
\end{gathered}
$$

onde $v$ é o custo total do investimento; $c_{i j}$ é o custo de adição de uma linha no caminho $i-j ; S$ é a matriz de incidência nó-ramo transposta; $\theta_{j}$ é o ângulo de tensão na barra $j ; \gamma_{i j}$ é o valor da susceptância da linha adicionada em $i-j ; f_{i j}$ é o fluxo de potência ativa no ramo $i-j ; \bar{f}_{i j}$ é o limite superior de $f_{i j} ; g$ é o vetor de gerações; $\bar{g}$ é o vetor com o limite superior das gerações; $d$ é o vetor com o valor das cargas (demandas) do sistema; $n_{i j}$ representa o número de linhas adicionadas no caminho $i-j ; \bar{n}_{i j}$ é o número máximo de adição de linhas no ramo $i-j ; n_{i j}^{0}$ é número de linhas presentes na topologia base no caminho $i-j$; $\Omega$ é o conjunto de todos os ramos.

A Equação (2) representa a equação de balanço de potência em cada nó. Essa restrição corresponde à lei das correntes de Kirchhoff. A equação (3) modela a lei das tensões de Kirchhoff. A equação (3) é não-linear devido ao produto $\left(n_{i j}^{0}+n_{i j}\right)\left(\theta_{i}-\theta_{j}\right)$. As demais restrições estão relacionadas aos limites das variáveis. Este modelo é considerado um modelo de programação não-linear inteiro-misto.

Para contornar a não-linearidade, costuma-se utilizar o modelo cc com a relaxação linear proposta por Villasana- 
Garver-Salon (Villasana et al., 1985). O modelo decorrente da relaxação linear do modelo cc é chamado modelo híbrido linear e será utilizado neste trabalho.

A relaxação proposta em (Villasana et al., 1985) consiste em considerar o conjunto de restrições (3) apenas para as linhas já existentes no sistema. Esta relaxação tem grande importância prática, uma vez que quando usada em um método construtivo, encontra solução factível para o modelo cc. Porém, quando aplicado um método exato, isto pode não acontecer. Mesmo a relaxação proposta apresenta grande dificuldade de convergência quando se lida com sistemas de grande porte.

O modelo proposto em (Villasana et al., 1985) pode ser representado de acordo com as Equações. (9-17).

$$
\min v=\sum_{(i, j) \in \Omega} c_{i j} n_{i j}
$$

s.a.

$$
\begin{gathered}
S f+S_{0} f_{0}+g=d \\
f_{i j}^{0}-\gamma_{i j} n_{i j}^{0}\left(\theta_{i}-\theta_{j}\right)=0 \forall(i, j) \in \Omega_{1} \\
\left|f_{i j}^{0}\right| \leq n_{i j}^{0} \bar{f}_{i j} \forall(i, j) \in \Omega_{1} \\
\left|f_{i j}^{\prime}\right| \leq n_{i j} \bar{f}_{i j} \forall(i, j) \in \Omega \\
0 \leq g \leq \bar{g} \\
0 \leq n_{i j} \leq \bar{n}_{i j} \\
n_{i j} \text { inteiro } \forall(i, j) ; f_{i j} \text { irrestrito } \forall(i, j) ; \\
\theta_{j} \text { irrestrito } \forall j
\end{gathered}
$$

$S_{0}$ representa a matriz de incidência nó-ramo transposta das linhas existentes na configuração base; $f_{0}$ representa o vetor de fluxos nas linhas existentes na configuração base, cujos elementos são $f_{i j}^{0}$; $\Omega$ é o conjunto de todas as linhas possíveis de serem adicionadas ao sistemas e $\Omega_{1}$ é o conjunto das linhas pertencentes à configuração base. As demais variáveis são as definidas no modelo cc anterior.

\section{ALGORITMO BRANCH AND CUT}

$\mathrm{O}$ algoritmo branch and cut $(\mathrm{B} \& \mathrm{C})$ é um método exato de programação linear inteira introduzida em (Padberg and Rinaldi, 1987; Padberg and Rinaldi, 1991) aplicado inicialmente para solução do problema do caixeiro viajante.

Nesse algoritmo, a relaxação linear do problema é restringida adicionando um conjunto de desigualdades válidas à formulação (Nemhauser and Wolsey, 1999; Balas et al., 1996).

$\mathrm{Na}$ implementação proposta neste trabalho, a técnica pode ser resumida em adicionar cortes válidos ao modelo inicial nos subproblemas gerados pela estrutura branch and bound, proposta por (Land and Doig, 1960) usando o critério de separação introduzido em (Benichou et al., 1971), (Gauthier and Ribiere, 1977) e (Linderoth and Savelsbergh, 1999). Neste artigo, seguindo a estratégia sugerida por (Cornuéjols, 2008), aplicam-se cortes singulares ao PEST em vez dos cortes genéricos sugeridos na literatura.

\subsection{Inequações válidas para o PEST}

Nesta seção serão apresentadas as inequações utilizadas na abordagem B\&C proposta neste trabalho para solução do PEST. As seguintes notações e símbolos serão adotadas para apresentação das inequações válidas para o PEST:

- $\Omega_{2}$ é o conjunto de todas as barras do sistema.

- $\mathbb{V}(k)$ é o conjunto de todas as linhas (arcos) ligadas à barra $k$.

- $\mathbb{N}(k)$ é o conjunto de todas as barras (nós) ligadas à barra $k$. A barra $i \in \mathbb{N}(k)$ será indexada como $\mathbb{N}(k)_{i}$.

- $N D G$ é o conjunto de todas as barras que são tanto geração como demanda.

- $N O D$ é o conjunto de todas as barras que são apenas demanda.

\subsubsection{Cortes nas barras de demanda}

O primeiro tipo de inequação ou corte válido que apresentaremos neste artigo parte do princípio de que a quantidade de linhas instaladas que se conectam a uma determinada barra deve proporcionar uma capacidade de transmissão maior ou igual à demanda prevista nessa barra, conforme a Equação (18).

$$
\sum_{(i, j) \in \mathbb{V}(k)}\left(n_{i j}^{o}+n_{i j}\right) \bar{f}_{i j}+g_{k} \geq d_{k} \forall k \in N D G
$$

Por redução de coeficientes ((Wolsey, 1998) e (Dietrich and Escudero, 1990)), a Equação (18) é equivalente à Equação (19).

$$
\sum_{(i, j) \in \mathbb{V}(k)} \min \left\{b, \bar{f}_{i j}\right\} n_{i j}+g_{k} \geq b \forall k \in N D G
$$

onde $b=d_{k}-\sum_{(i, j) \in \mathbb{V}(k)} n_{i j}^{o} \bar{f}_{i j}$. Por equivalente, quer-se dizer que (18) e (19) têm as mesmas soluções não-negativas $\operatorname{com} n_{i j}$ inteiro e não negativo. 
Um corte mais restritivo pode ser adicionado ao problema quando se considera as barras que são apenas de demanda, conforme a Equação (20).

$$
\sum_{(i, j) \in \mathbb{V}(k)}\left(n_{i j}^{o}+n_{i j}\right) \bar{f}_{i j} \geq d_{k} \forall k \in N O D
$$

Uma inequação válida pode ser gerada a partir da Equação (20). Para tanto, seja $\Gamma=\mathbb{V}(k)-\{l k\}$ para algum $l k \in$ $\mathbb{V}(k)$. A Equação (20) pode ser escrita como a Equação (21).

$$
\left(n_{l k}^{o}+n_{l k}\right) \bar{f}_{l k}+\sum_{(i, j) \in \Gamma}\left(n_{i j}^{o}+n_{i j}\right) \bar{f}_{i j} \geq d_{k} \forall k \in N O D
$$

dividindo ambos os lados da Equação (21) por $\bar{f}_{l k}$, obtém-se $(\forall k \in N O D)$.

$$
n_{l k}+\frac{\sum_{(i, j) \in \Gamma} n_{i j} \bar{f}_{i j}}{\bar{f}_{l k}} \geq \frac{d_{k}-\sum_{(i, j) \in \Gamma} n_{i j}^{o} \bar{f}_{i j}}{\bar{f}_{l k}}-n_{l k}^{o} .
$$

Uma vez que

$$
\sum_{(i, j) \in \Gamma} n_{i j}\left\lceil\frac{\bar{f}_{i j}}{\bar{f}_{l k}}\right\rceil \geq \frac{\sum_{(i, j) \in \Gamma} n_{i j} \bar{f}_{i j}}{\bar{f}_{l k}}
$$

então a Equação (24) é valida.

$$
n_{l k}+\sum_{(i, j) \in \Gamma} n_{i j}\left\lceil\left[\begin{array}{l}
\bar{f}_{i j} \\
\overline{\bar{f}_{l k}}
\end{array}\right] \geq \frac{d_{k}-\sum_{(i, j) \in \Gamma} n_{i j}^{o} \bar{f}_{i j}}{\bar{f}_{l k}}-n_{l k}^{o}\right.
$$

Como o lado esquerdo da Equação (24) tem todos os seus coeficientes inteiros e $n_{i j}$ é inteiro, o lado esquerdo da Equação (24) deve ser inteiro. Arredondando o lado direito irá resultar em uma inequação válida (procedimento ChvátalGomory (Caprara and Fischetti, 1996)).

$$
n_{l k}+\sum_{(i, j) \in \Gamma} n_{i j}\left\lceil\frac{\bar{f}_{i j}}{\bar{f}_{l k}}\right\rceil \geq\left\lceil\frac{d_{k}-\sum_{(i, j) \in \Gamma} n_{i j}^{o} \bar{f}_{i j}}{\bar{f}_{l k}}\right\rceil-n_{l k}^{o}
$$

Dado o modelo representado pelas Equações (9)-(17), uma outra forma de gerar cortes nas barras de carga é considerando a Equação (10), sem perda de generalização. A Equação (26) expressa uma linha da matriz de igualdades do conjunto de restrições referentes à Equação (10).

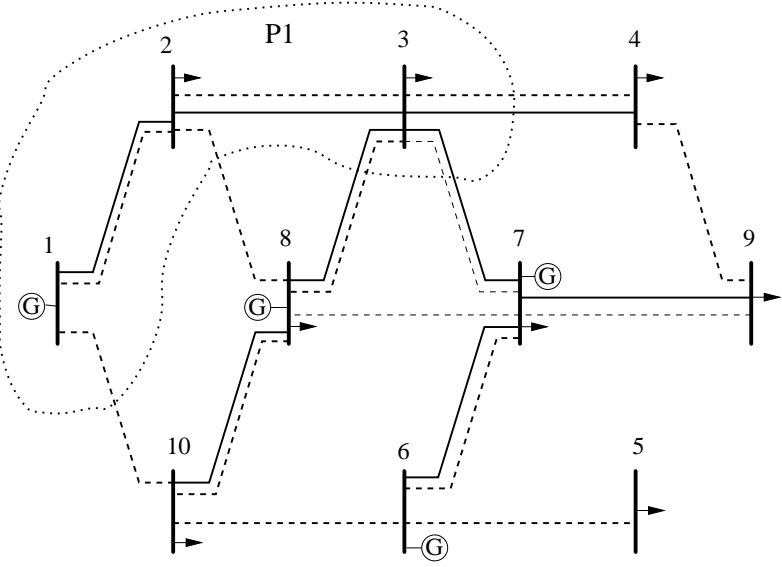

Figura 1: Sistema fictício de 10 barras

$\sum_{(i, j) \in \mathbb{V}(k)} s_{i j} f_{i j}^{\prime}+\sum_{(i, j) \in \mathbb{V}(k)} s_{i j}^{0} f_{i j}^{0}+g_{k}=d_{k} \forall k \in \Omega_{2}$

Desde que $f_{i j}^{\prime} \leq n_{i j} \bar{f}_{i j}$ e $s_{i j} \in\{-1,0,1\}$, então

$$
n_{i j} \bar{f}_{i j} \geq s_{i j} f_{i j}^{\prime} \forall(i, j) \in \Omega
$$

Assim, a Equação (28) é válida.

$$
\sum_{(i, j) \in \mathbb{V}(k)} n_{i j} \bar{f}_{i j}+\sum_{(i, j) \in \mathbb{V}(k)} s_{i j}^{0} f_{i j}^{0}+g_{k} \geq d_{k} \forall k \in \Omega_{2}
$$

\subsubsection{Inequações cutset}

O sistema fictício da Figura 1 pode ser representado por um grafo $G=(N, V)$ onde $N$ é o conjunto de nós (barras do sistema) e $V$ é o conjunto de arcos (linhas do sistema).

Seja $S \subset N$ um conjunto não vazio de $N$ e $\bar{S}=N \backslash S$ o seu complemento. $(S, \bar{S})$ são inequações de separação de conjunto, ou simplesmente, um custset $^{1}$, ou seja, um conjunto de arcos que conectam um nó $S$ a um nó $\bar{S}$ (Costa et al., 2009b).

Uma forma de gerar inequações cutset para o sistema da Figura 1 é considerando caminhos no grafo correspondente. Assim, seja $P=\left(N_{p}, V_{p}\right)$ um subgrafo de $G$ representando um caminho naquele grafo. Seja também $B=$ $\left(\cup_{k \in N_{p}} \mathbb{V}(k)\right) \backslash V_{p}$ o conjunto de todos os arcos conectados aos nós pertencentes ao caminho $P$ e não pertencentes ao conjunto $V_{p}$. A Equação (29) é válida.

\footnotetext{
${ }^{1}$ Preferimos utilizar o termo em inglês em razão de seu amplo uso na literatura técnica.
} 


$$
\sum_{(i, j) \in B}\left(n_{i j}^{o}+n_{i j}\right) \bar{f}_{i j}+\sum_{k \in N_{p}} g_{k} \geq \sum_{k \in N_{p}} d_{k}
$$

Exemplo 1 Para o caminho $P 1$, destacado na Figura 1, a seguinte inequação pode ser gerada.

$$
\begin{aligned}
n_{1-10} \bar{f}_{1-10}+ & \left(1+n_{3-4}\right) \bar{f}_{3-4}+\left(1+n_{3-7}\right) \bar{f}_{3-7} \\
& +\left(1+n_{3-8}\right) \bar{f}_{3-8}+g_{1} \geq d_{2}+d_{3}
\end{aligned}
$$

A Equação (30) é uma generalização da Equação (18), indicando-se que a capacidade de transmissão é suficiente para atendimento das cargas existentes no caminho. Uma variação importante de (30) surge quando um caminho é composto somente por barras de carga, como o caminho $P 2$, destacado na Figura 2.

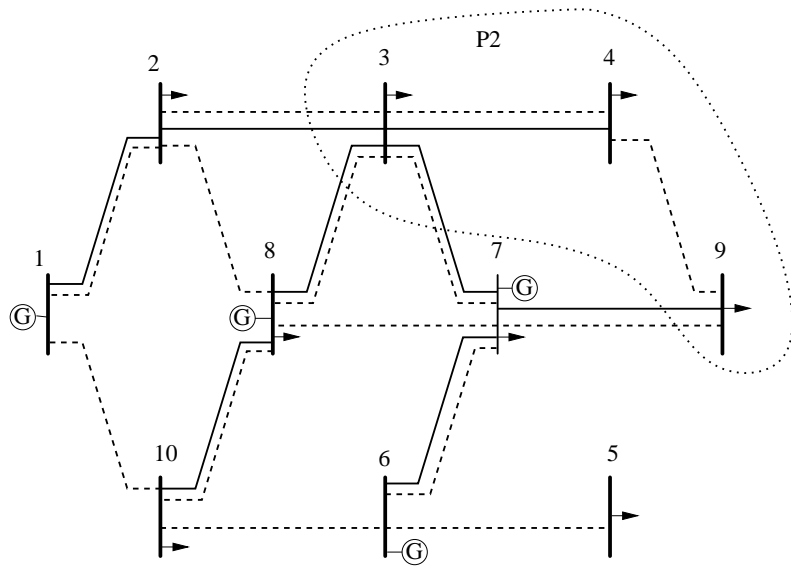

Figura 2: Sistema de 10 barras: caminho com barras de carga apenas.

A parte $\sum_{k \in N_{p}} g_{k}$ da Equação (29) é desnecessária para o caminho $P 2$. Portanto, a desigualdade da Equação (31) é válida para os caminhos envolvendo somente barras de carga.

$$
\sum_{(i, j) \in B}\left(n_{i j}^{o}+n_{i j}\right) \bar{f}_{i j} \geq \sum_{k \in N_{p}} d_{k}
$$

É possível obter uma desigualdade mais forte a partir da Equação (31), por arredondamento. Seja um $\bar{f}_{l k}$, para algum $l k \in B$, e seja também $L=B-\{l k\}$. Assim como a Equação (24), a desigualdade Equação (32) é válida.

$$
\begin{aligned}
& n_{l k}+\sum_{(i, j) \in L} n_{i j}\left\lceil\frac{\bar{f}_{i j}}{\bar{f}_{l k}}\right\rceil \geq \\
&\left\lceil\frac{\sum_{k \in N_{p}} d_{k}-\sum_{(i, j) \in L} n_{i j}^{o} \bar{f}_{i j}}{\bar{f}_{l k}}\right\rceil-n_{l k}^{o}
\end{aligned}
$$

É importante destacar que, como observado na Figura 1, a geração de todas as desigualdades do tipo Equação (29) resulta em um problema do tipo combinatório. Portanto, propõe-se uma heurística para definir qual ramo (variável $n_{i j}$ ) selecionar para construir as inequações de cutset. Neste caso, a heurística é guiada pela a execução do algoritmo B\&B da seguinte forma: resolve-se o modelo representado pelas Equações (9-17) relaxando-se as restrições de integralidade. Havendo variáveis $n_{i j}$ de valor não inteiro, deve-se selecionar uma para dividir o problema. Uma vez definida a variável para dividir, esta mesma variável é empregada para gerar as inequações de cutset. Portanto, propomos que sejam construídas inequações envolvendo as barras $i$ e $j$. Desta forma, além dos limitantes inseridos pelo algoritmo $\mathrm{B} \& \mathrm{~B}$ à variável $n_{i j}$ selecionada para dividir o problema, serão inseridas também as inequações de cutset envolvendo esta variável.

Assim, empregam-se as estratégias: selecionar e aplicar limitantes do algoritmo B\&B, juntamente com os cortes adicionados em cada iteração do processo de busca. Ou seja, o princípio básico do algoritmo branch and cut é aplicado.

\subsubsection{Melhorando a estabilidade do algoritmo}

Os modelos apresentados nas seções anteriores estão entre os mais usados pelos pesquisadores para solução do PEST. Para solução por programação linear inteira mista é importante que a relaxação linear do modelo seja o mais próxima de sua envoltória convexa (Nemhauser and Wolsey, 1999). Portanto, é importante aplicar cortes a priori ao modelo, deixando-o mais próximo da envoltória convexa. Para os modelos do PEST em que se considera a LTK, cortes podem ser obtidos a partir das Equações (11) e (12).

Considerando que pela Equação (11), $f_{i j}^{0}=\gamma_{i j} n_{i j}^{0}\left(\theta_{i}-\right.$ $\left.\theta_{j}\right) \forall(i, j) \in \Omega_{1}$. e substituindo $f_{i j}^{0}$ em (12), obtemos

$$
\left|\gamma_{i j} n_{i j}^{0}\left(\theta_{i}-\theta_{j}\right)\right| \leq n_{i j}^{0} \bar{f}_{i j} \forall(i, j) \in \Omega_{1}
$$

e, consequentemente,

$$
\left|\theta_{i}-\theta_{j}\right| \leq \frac{\bar{f}_{i j}}{\gamma_{i j}} \forall(i, j) \in \Omega_{1}
$$


Com o uso da restrição anterior, observamos uma melhora no tempo computacional em relação à convergência do problema de programação linear (PPL) resolvido durante o processo de resolução. Esta estratégia auxilia solvers como o MINOS utilizado neste trabalho. Uma outra estratégia comumente adotada é a adoção de ângulo zero na barra de referência. Embora matematicamente não seja necessário, essa prática resulta na melhoria da resolução.

\subsection{Modelo híbrido linear com as inequa- ções propostas}

As Equações (35-49) modelam o PEST considerando as inequações propostas neste trabalho, para um conjunto $P$ de caminhos e para todo conjunto $P O D$ de caminhos envolvendo apenas barras de demanda. Em cada nó do B\&C deve-se resolver o seguinte modelo.

$$
\min v=\sum_{(i, j) \in \Omega} c_{i j} n_{i j}
$$

s.a

$$
\begin{aligned}
& \text { Inequações nas barras de demanda: } \\
& \sum_{(i, j) \in \mathbb{V}(k)} \min \left\{b, \bar{f}_{i j}\right\} n_{i j}+g_{k} \geq b \forall k \in N D G(36) \\
& n_{l k}+\sum_{(i, j) \in \Gamma} n_{i j}\left\lceil\bar{f}_{i j}\right\rceil \geq \\
& \left\lceil\frac{d_{k}-\sum_{(i, j) \in \Gamma} n_{i j}^{o} \bar{f}_{i j}}{\bar{f}_{l k}}\right\rceil-n_{l k}^{o} \\
& \sum_{(i, j) \in \mathbb{V}(k)} n_{i j} \bar{f}_{i j}+\sum_{(i, j) \in \mathbb{V}(k)} s_{i j}^{0} f_{i j}^{0}+ \\
& g_{k} \geq d_{k} \forall k \in \Omega_{2} \\
& \sum_{(i, j) \in B}\left(n_{i j}^{o}+n_{i j}\right) \bar{f}_{i j}+ \\
& \sum_{k \in N_{p}} g_{k} \geq \sum_{k \in N_{p}} d_{k} \\
& n_{l k}+\sum_{(i, j) \in L} n_{i j}\left\lceil\frac{\bar{f}_{i j}}{\bar{f}_{l k}}\right\rceil \\
& \geq\left\lceil\frac{\sum_{k \in N_{P O D}} d_{k}-\sum_{(i, j) \in L} n_{i j}^{o} \bar{f}_{i j}}{\bar{f}_{l k}}\right\rceil-n_{l k}^{o}
\end{aligned}
$$

Modelo clássico de (Villasana et al., 1985):

$$
\begin{gathered}
S f+S_{0} f_{0}+g=d \\
f_{i j}^{0}-\gamma_{i j}\left(\theta_{i}-\theta_{j}\right)=0 \forall(i, j) \in \Omega_{1} \\
\left|\theta_{i}-\theta_{j}\right| \leq \frac{\bar{f}_{i j}}{\gamma_{i j}} \forall(i, j) \in \Omega_{1} \\
\left|f_{i j}^{0}\right| \leq n_{i j}^{0} \bar{f}_{i j} \forall(i, j) \in \Omega_{1} \\
\left|f_{i j}^{\prime}\right| \leq n_{i j} \bar{f}_{i j} \forall(i, j) \in \Omega \\
0 \leq g \leq \bar{g} \\
0 \leq n_{i j} \leq \bar{n}_{i j} \\
n_{i j} \text { inteiro } \forall(i, j) ; f_{i j} \text { irrestrito } \forall(i, j) ; \\
\theta_{j} \text { irrestrito } \forall j
\end{gathered}
$$

Vale destacar que todas as desigualdades de cutset e as envolvendo as barras de demanda propostas neste artigo são válidas para todos os modelos do PEST, tanto linear como não-linear.

\subsection{Algoritmo proposto}

$\mathrm{Na}$ abordagem clássica do algoritmo $\mathrm{B} \& \mathrm{C}$, os cortes adicionados são os cortes genéricos propostos na literatura além de cortes específicos a cada problema. Na presente proposta, não usaremos o algoritmo $\mathrm{B} \& \mathrm{C}$ básico nem os cortes clássicos para programação inteira mista (Nemhauser and Wolsey, 1999; Balas et al., 1996). Em vez disso, e para verificar a qualidade dos cortes propostos pelos autores, usaremos apenas os cortes apresentados neste trabalho (Equações (19), (25) e (34)) e as inequações de cutset. A Figura 6 apresenta o fluxograma do algoritmo branch and cut proposto.

\section{ALGORITMO PARA CONSTRUÇÃO DAS INEQUAÇÕES CUTSET}

Nesta seção detalharemos as principais estruturas de dados e algoritmos usados na implementação computacional do método proposto neste trabalho.

\subsection{Fundamentos}

Toda e qualquer implementação computacional eficiente parte da escolha correta das estruturas de dados a serem utilizadas durante a programação. Nesta seção sugerimos algumas estruturas de dados que podem ser utilizadas para gerar caminhos em um grafo de forma automática. A Figura 3 mostra a representação gráfica do tipo vizinho.

É importante ressaltar que para evitar um esforço computacional muito elevado construindo os caminhos nos grafos, deve-se evitar adicionar cortes envolvendo poucos nós. Nesta proposta, o parâmetro $\Psi$ foi utilizado para indicar o número 


$$
\begin{array}{|l|}
\hline \text { Linha } \\
\hline \text { Barra } \\
\hline
\end{array}
$$

Figura 3: Representação do tipo de dados vizinho.

máximo de nós que devem estar envolvidos nos caminhos gerados. O número mínimo adotado é 2. Assim, para todo caminho construindo $P=(N, V), 2 \leq|N| \leq \Psi$. As figuras 4 e 5 mostram árvores que representam a vizinhança das barras 1 e $2, \operatorname{com} \Psi=4$, respectivamente.

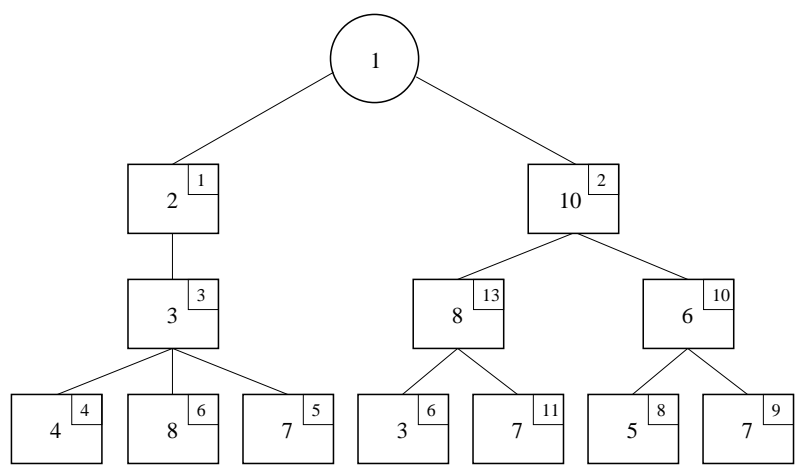

Figura 4: Árvore com a vizinhança da barra 1.

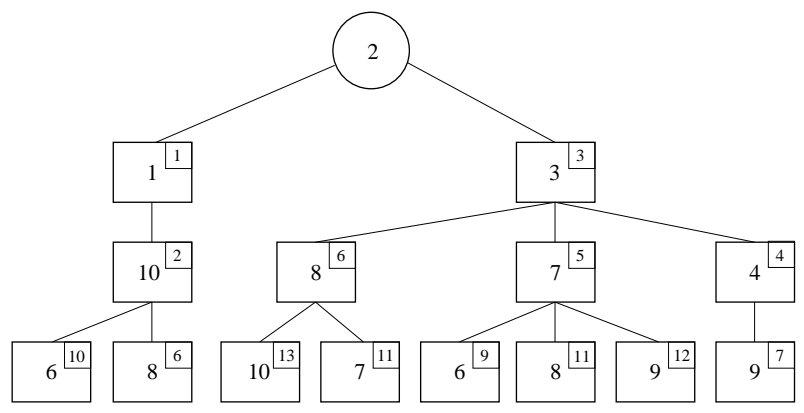

Figura 5: Árvore com a vizinhança da barra 2.

Não é necessário construir todos os caminhos do grafo a priori. $\mathrm{O}$ algoritmo $\mathrm{B} \& \mathrm{C}$ sugerido neste trabalho é guiado pela execução do algoritmo B\&B para construir os caminhos, e assim, gerar as inequações de cutset. Por exemplo, após resolver o PPL correspondente ao modelo de otimização relaxado, se $n_{1-2}$ não for inteiro, deve-se construir caminhos envolvendo esta linha. Isto é, visitando nós a partir de 1 para 2 da Figura 4, e a partir de 2 para 1 da Figura 5. Isto significa que somente é necessário construir cortes envolvendo as barras 1 e 2 . Exemplo de caminhos envolvendo a linha $n_{1-2}$ são: $\{1 \rightarrow 2 ; 1 \rightarrow 2 \rightarrow 3 ; 1 \rightarrow 2 \rightarrow 3 \rightarrow 7 ; \ldots ; 2 \rightarrow 1 \rightarrow 10 ; \ldots\}$. Se após aplicar os cortes gerados ao modelo, a solução encontrar a solução ótima, não será mais necessário gerar novos caminhos.
Sugerimos construir todos os cortes envolvendo a variável $n_{i j}$ não inteira e selecionada para dividir o problema, e armazenar estes cortes em uma lista de cortes para que todos os nós da árvore B\&B possam usá-los.

Durante a execução do algoritmo B\&C podem ser gerados cortes iguais. Para evitar que isto ocorra, sugerimos que se mantenha uma lista de cortes válidos já gerados e que um novo corte seja adicionado a esta lista se e somente se o novo corte não for paralelo a nenhum outro corte na lista.

Definição $1 \mathrm{O}$ paralelismo entre dois cortes, $\alpha x \leq \alpha_{0}$ e $\beta x \leq \beta_{0}$, é a medida do cosseno do ângulo entre os dois vetores normais a cada plano e é dado por

$$
\operatorname{par}(\alpha, \beta)=\frac{\alpha^{T} \beta}{\|\alpha\|\|\beta\|},
$$

de forma que dois vetores paralelos têm $\operatorname{par}(\alpha, \beta)=1$ (Andreello et al., 2007).

Neste trabalho, como sugerido em (Andreello et al., 2007), um novo corte $\alpha$ será descartado se $\operatorname{par}(\alpha, \beta) \geq \max _{\text {par }}$ para algum $\beta$ pertencente à lista de cortes. Sugerimos o algoritmo recursivo 1 para construir todos os caminhos $P=$ $(N, V), 2 \leq|N| \leq \Psi$ a partir da barra de para a barra para.

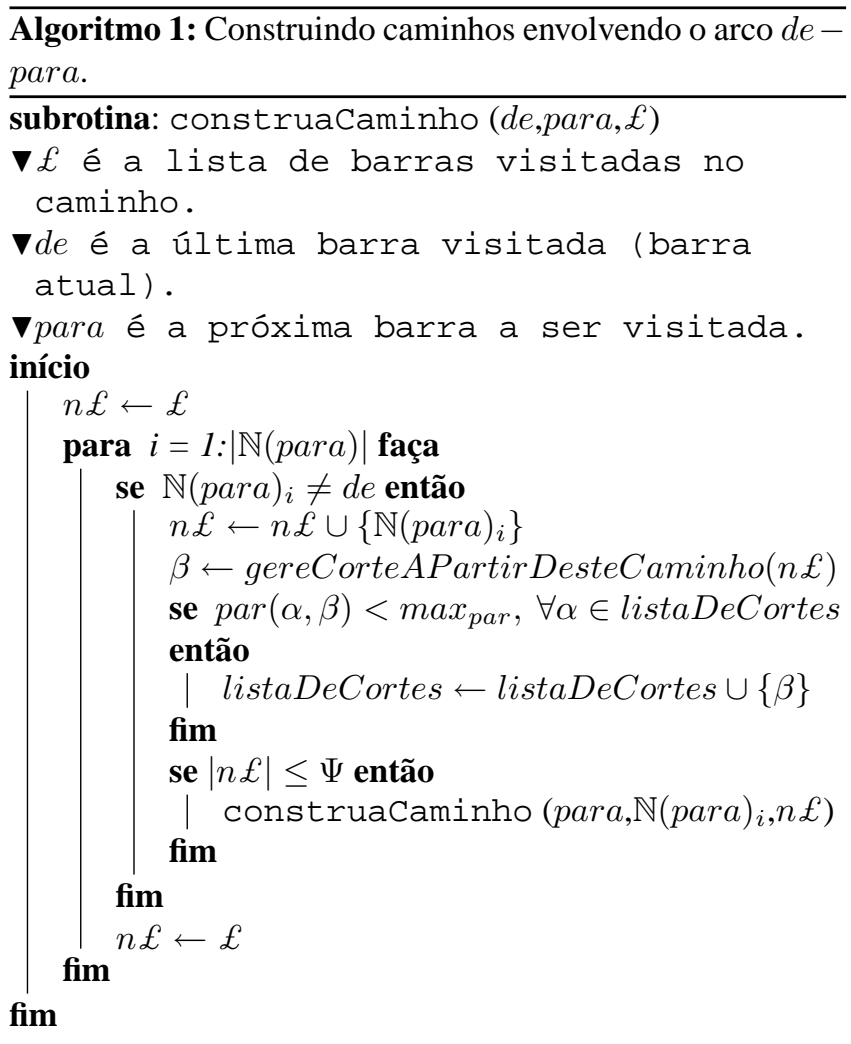




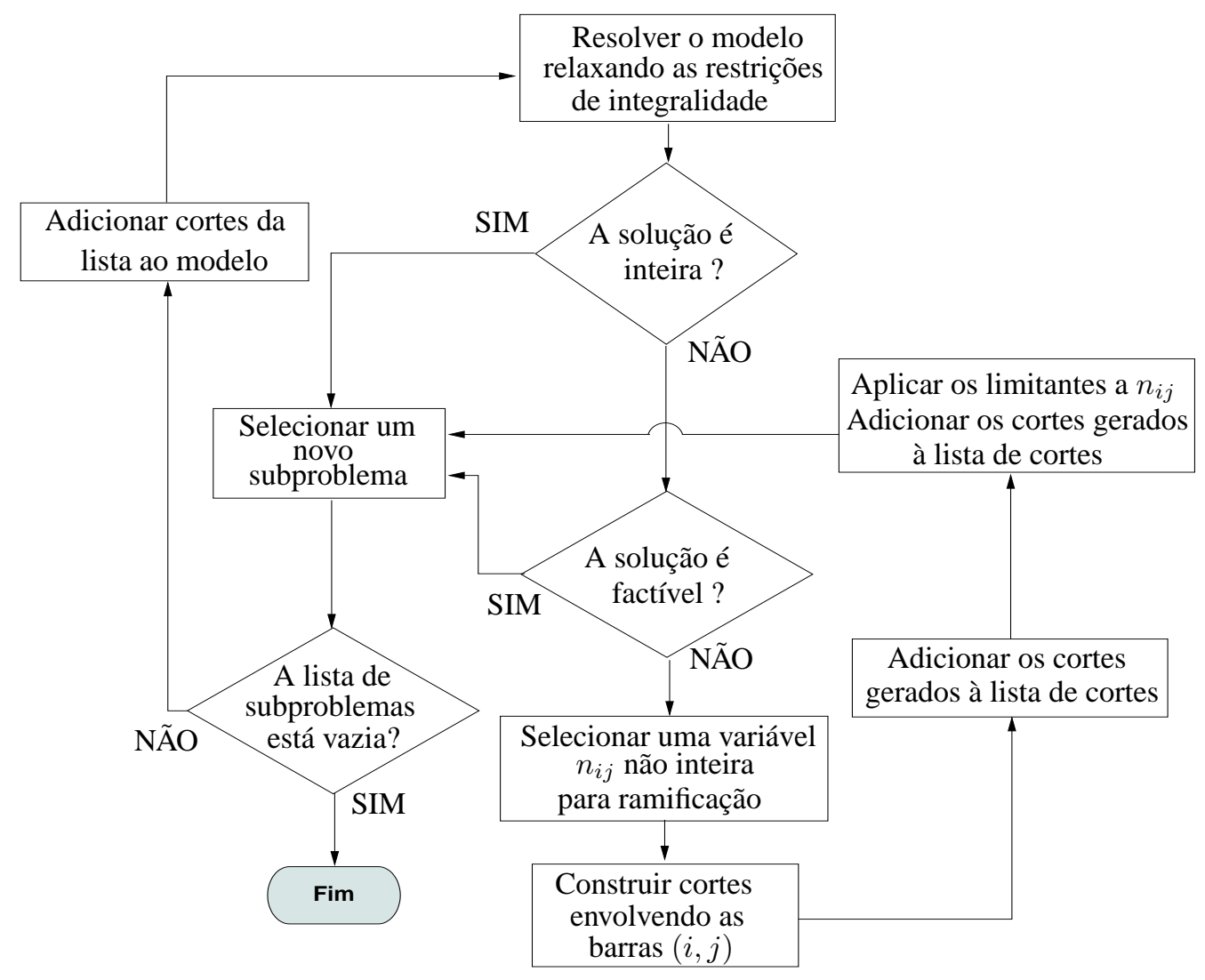

Figura 6: Diagrama do algoritmo branch and cut proposto. 


\section{TESTES COMPUTACIONAIS}

Nesta seção serão descritos os sistemas teste e os resultados obtidos ao aplicar as inequações propostas. Uma comparação com o algoritmo B\&B sem as inequações propostas também será apresentada.

A implementação computacional foi desenvolvida pelos autores na linguagem Fortran 90 e compilada com o compilador Intel(R) Fortran Compiler 10.1.024 [IA-32]. Foi utilizado o pacote computacional MINOS 5.4 (desenvolvido em Fortran 77) (Murtagh and Saunders, 1987) para resolver os PPLs necessários. Todos os testes foram realizados em um computador com processador Intel ${ }^{(R)} \operatorname{Core}^{T M} 2 \mathrm{CPU} 6400$ @ $2.13 \mathrm{GHz}$ 2.13GHz, 3,00 GB de RAM; sistema operacional Windows XP.

Para todos os sistemas, ao parâmetro $\max _{\text {par }}$ foi atribuído o valor $0,99\left(\max _{p a r}=0,99\right)$. Ao parâmetro $\Psi$ foram atribuídos valores diferentes dependendo do sistema, como mostrado na Tabela 1.

\subsection{Testes com o sistema colombiano}

Foram realizados testes com os planos 2002 (Col02) e 2012 (Col12). O sistema Col02 é composto por 93 barras, 155 linhas e $9.750 \mathrm{MW}$ de demanda e geração. O Col12 é composto por 93 barras, 155 linhas e $14.559 \mathrm{MW}$ de demanda e geração.

Para o plano Col02, a solução ótima encontrada sugere um investimento de $v=$ US\$232.313.000 com 8 linhas adicionadas em 5 ramos e a seguinte configuração:

$$
\begin{gathered}
n_{52-88}=1 ; n_{43-88}=2 ; n_{57-81}=1 ; n_{09-69}=3 ; \\
n_{50-54}=1 .
\end{gathered}
$$

Para o plano Col12, a solução ótima sugere um investimento de $v=$ US\$ 470.361 .000 com 26 linhas adicionadas em 19 ramos e a seguinte configuração:

$$
\begin{gathered}
n_{52-88}=1 ; n_{43-88}=2 ; n_{57-81}=1 ; n_{14-31}=1 ; \\
n_{15-18}=1 ; n_{55-84}=1 ; n_{55-62}=1 ; n_{69-70}=1 ; \\
n_{9-69}=1 ; n_{60-69}=3 ; n_{31-72}=3 ; n_{19-22}=1 ; \\
n_{5-6}=1 ; n_{19-58}=1 ; n_{27-64}=1 ; n_{19-66}=3 ; \\
n_{34-70}=1 ; n_{50-54}=1 ; n_{68-86}=1 .
\end{gathered}
$$

\subsection{Testes com o sistema Sul Brasileiro}

O sistema sul brasileiro apresenta 46 barras, 79 linhas e 6.880 MW de demanda. Para este sistema, existem dois planos de expansão: com redespacho da geração (10.545 MW de geração) (Sul1) e sem redespacho da geração (6.880 MW de geração) (Sul2).
Para o sistema Sul1, a solução ótima sugere um investimento de $v=\mathrm{US} \$ 63.163 .000,00$ com 7 linhas adicionadas em 5 ramos e a seguinte configuração:

$$
\begin{gathered}
n_{20-23}=1 ; n_{20-21}=2 ; n_{42-43}=1 ; n_{46-06}= \\
1 ; n_{05-06}=2 .
\end{gathered}
$$

Para o sistema Sul2, a solução ótima sugere um investimento de $v=\mathrm{US} \$ 141.350 .000,00$ com 14 linhas adicionadas em 11 ramos e a seguinte configuração:

$$
\begin{gathered}
n_{20-21}=1 ; n_{42-43}=2 ; n_{46-06}=1 ; n_{25-32}=1 ; \\
n_{31-32}=1 ; n_{28-31}=1 ; n_{28-30}=1 ; n_{26-29}=2 ; \\
n_{24-25}=2 ; n_{29-30}=1 ; n_{05-06}=1 .
\end{gathered}
$$

\subsection{Teste com o sistema Norte-Nordeste brasileiro}

$\mathrm{O}$ sistema norte-nordeste brasileiro (NN) possui 87 barras, 183 linhas e $20.316 \mathrm{MW}$ de demanda e 20.316,51 MW de geração. Este sistema é muito complexo e sua solução ótima para o modelo híbrido era desconhecida até o presente trabalho (Romero et al., 2002), (Romero et al., 2007).

Para o sistema NN a solução ótima encontrada sugere um investimento de $v=\mathrm{US} \$ 1.260 .567 .000,00$ com 65 linhas adicionadas em 46 ramos e a seguinte configuração:

$n_{2-60}=2 ; n_{5-58}=2 ; n_{5-60}=2 ; n_{5-68}=1 ; n_{8-17}=1 ;$
$n_{8-62}=2 ; n_{9-10}=1 ; n_{10-11}=1 ; n_{11-12}=1 ;$
$n_{12-15}=1 ; n_{12-17}=1 ; n_{3-14}=1 ; n_{3-15}=1 ;$
$n_{14-59}=1 ; n_{15-16}=1 ; n_{16-44}=2 ; n_{17-18}=2 ;$
$n_{8-50}=6 ; n_{19-20}=1 ; n_{20-21}=1 ; n_{20-21}=1 ;$
$n_{20-38}=1 ; n_{22-58}=1 ; n_{24-43}=1 ; n_{25-55}=1 ;$
$n_{26-29}=2 ; n_{26-54}=2 ; n_{29-30}=2 ; n_{30-31}=1 ;$
$n_{34-41}=2 ; n_{36-46}=1 ; n_{39-86}=1 ; n_{40-45}=1 ;$
$n_{43-55}=1 ; n_{43-58}=1 ; n_{44-46}=1 ; n_{48-49}=2 ;$
$n_{49-50}=1 ; n_{52-59}=1 ; n_{53-86}=1 ; n_{54-58}=1 ;$
$n_{61-85}=3 ; n_{61-86}=1 ; n_{62-67}=2 ; n_{67-69}=1 ;$
$n_{69-87}=1$.

\section{ANÁLISE DOS RESULTADOS}

A Tabela 1 apresenta um resumo dos resultados obtidos através da aplicação da abordagem proposta neste trabalho. A Tabela 2 apresenta os resultados da aplicação do algoritmo $\mathrm{B} \& \mathrm{~B}$. A implementação do algoritmo $\mathrm{B} \& \mathrm{~B}$ é a mesma para a abordagem do algoritmo $B \& C$, porém sem os cortes propostos neste trabalho.

Nas tabelas 1 e 2, a coluna PL2OptBF apresenta o número de PPLs executados até se encontrar a solução; a coluna 
PL2Conv apresenta o número de PPLs executados até a convergência total do algoritmo; a coluna Tempo mostra o tempo em segundos passados durante a execução do algoritmo e a coluna MNS mostra o maior número de subproblemas armazenados durante a execução do algoritmo. $N C$ significa que o algoritmo não convergiu após executar mais de 15.000.000 PPLs ou atingiu o limite máximo de subproblemas armazenados. ( $M M N A \geq 200.000)$.

Tabela 1: Resumo dos resultados obtidos pelo método B\&C.

\begin{tabular}{crrrrr}
\hline Sistema & PL2OptBF & PL2Conv & Tempo & MNS & $\Psi$ \\
\hline SUL1 & 137 & 191 & 0,19 & 35 & 3 \\
SUL2 & 1.620 & 2.778 & 2,54 & 145 & 3 \\
COL02 & 972 & 1.341 & 2,32 & 70 & 3 \\
COL12 & 21.100 & 58.178 & 136,1 & 3.546 & 4 \\
NN & 1.062 .554 & 1.680 .080 & $13.132,3$ & 143.357 & 6 \\
\hline \hline
\end{tabular}

Tabela 2: Resumo dos resultados obtidos pelo método B\&B.

\begin{tabular}{crrrr}
\hline Sistema & PL2OptBF & PL2Conv & Tempo & $M N S$ \\
\hline SUL1 & 143 & 234 & 0,17 & 35 \\
SUL2 & 4.184 & 4,606 & 3,67 & 172 \\
COL02 & 1.667 & 2.156 & 3,43 & 166 \\
COL12 & 11.676 & 66.011 & 149,67 & 1.151 \\
NN & $N C$ & $N C$ & $N C$ & $N C$ \\
\hline \hline
\end{tabular}

Na Tabela 3 apresentamos uma comparação percentual entre os valores referentes à Tabela 1 e à Tabela 2 . O números em destaque na Tabela 3 indicam o percentual de melhoria do algoritmo B\&C em relação ao algoritmo B\&B. Destacamos que, pela Tabela 3, para o sistema COL02, o algoritmo $\mathrm{B} \& \mathrm{C}$ obteve convergência em tempo $32,36 \%$ menor do que o tempo do algoritmo B\&B.

Tabela 3: Diferença percentual do algoritmo branch and cut sobre o branch and bound.

\begin{tabular}{lrrrr}
\hline Sistema & $P L 2 O p t B F \%$ & $P L 2 C o n v \%$ & Tempo\% & $M N S \%$ \\
\hline SUL1 & $\mathbf{4 , 1 9}$ & $\mathbf{1 8}, \mathbf{3 8}$ & 10,53 & 0 \\
SUL2 & $\mathbf{6 1 , 2 8}$ & $\mathbf{3 9 , 6 9}$ & $\mathbf{3 0 , 7 9}$ & $\mathbf{1 5 , 7}$ \\
COL02 & $\mathbf{4 1 , 6 9}$ & $\mathbf{3 7 , 8}$ & $\mathbf{3 2}, \mathbf{3 6}$ & $\mathbf{5 7 , 8 3}$ \\
COL12 & 44,66 & $\mathbf{1 1 , 8 7}$ & $\mathbf{9 , 0 7}$ & 67,54 \\
\hline \hline
\end{tabular}

Como é possível observar nas tabelas anteriores, o método proposto convergiu para a solução ótima após resolver menos PPLs do que o algoritmo B\&B clássico.

Pode-se observar que o tempo computacional do algoritmo $\mathrm{B} \& \mathrm{C}$ foi maior do que o tempo computacional do algoritmo B\&B para o sistema SUL1, embora o algoritmo B\&C tenha resolvido menos PPLs. Uma explicação para isto se deve ao fato de o método B\&C aplicar alterações nos limitantes de algumas variáveis e adicionar novas restrições no modelo original a cada iteração. Enquanto que o algoritmo B\&B somente altera os limitantes das variáveis do modelo original. Desta forma, a solução dos PPLs do algoritmo B\&C será mais lenta do que a solução dos PPLs do algoritmo B\&B. Desta forma, o algoritmo $\mathrm{B} \& \mathrm{C}$ convergiu em menos iterações, porém cada iteração despendeu um tempo maior do que as iterações do B\&B. Casos assim são mais propensos em sistemas de pequeno porte já que a árvore B\&B para estes sistemas geralmente não cresce muito.

Outro ponto a ser destacado é o fato de o método B\&C proposto obter a solução do sistema NN. Esse sistema apresenta grande complexidade em função das seguintes características: elevada dimensão, presença de várias barras isoladas e pouca margem de manobra. A solução obtida é inédita na literatura técnica.

\section{CONCLUSÕES}

Neste artigo foi proposto um novo método B\&C aplicado ao PEST e novas inequações válidas para os modelos existentes para este problema. Com as inequações propostas foi possível reduzir o tempo de resolução em comparação ao algoritmo branch and bound clássico.

A estratégia de seleção das variáveis as quais originarão os cortes, evitam a geração indiscriminada de cortes e representa uma contribuição importante do método de resolução proposto.

As inequações apresentadas neste trabalho são válidas tanto para os modelos lineares como não lineares. Os resultados computacionais demonstraram a eficiência do método, tanto na redução do tempo de convergência como também a capacidade de obter a solução ótima para o sistema NN.

\section{AGRADECIMENTOS}

A pesquisa que originou este trabalho tem sido realizada com o apoio da FAPESP. Os autores agradecem ao editor associado e aos revisores por seus comentários e sugestões.

\section{REFERÊNCIAS}

Andreello, G., Caprara, A. and Fischetti, M. (2007). Embedding $\{0,1 / 2\}$-Cuts in a Branch-and-Cut Framework: A Computational Study, INFORMS Journal on Computing. 19(2): 229-238.

Asada, E. N., Carreno, E., Romero, R. and Garcia, A. V. (2006). A parallel branch and bound algorithm to transmission expansion planning, X SEPOPE, Florianópolis.

Balas, E., Ceria, S., Cornuéjols, G. and Natraj, N. (1996). Gomory cuts revisited, Operations Research Letters 19(1): 1-9. 
Benichou, M., Gauthier, J., Girodet, P., Hentges, G., Ribiere, G. and Vincent, O. (1971). Experiments in mixedinteger linear programming, Mathematical Programming 1(1): 76-94.

Binato, S., Oliveira, G. and Araujo, J. (2001). A greedy randomized adaptive search procedure for transmission expansion planning, IEEE Trans. Power Syst. 16(2): 247253.

Buygi, M., Balzer, G., Shanechi, H. and Shahidehpour, M. (2004). Market-based transmission expansion planning, IEEE Trans. Power Syst. 19(4): 2060-2067.

Caprara, A. and Fischetti, M. (1996). Chvátal-Gomory cuts, Mathematical Programming 74(3): 221-235.

Choi, J., El-Keib, A. and Tran, T. (2005). A fuzzy branch and bound-based transmission system expansion planning for the highest satisfaction level of the decision maker, IEEE Trans. Power Syst. 20(1): 476-484.

Chung, T., Li, K., Chen, G., Xie, J. and Tang, G. (2003). Multi-objective transmission network planning by a hybrid GA approach with fuzzy decision analysis, International Journal of Electrical Power and Energy Systems 25(3): 187-192.

Cornuéjols, G. (2008). Valid inequalities for mixed integer linear programs, Mathematical Programming 112(1): 344.

Costa, A. M., Cordeau, J.-F. and Gendron, B. (2009b). Benders, metric and cutset inequalities for multicommodity capacitated network design, Comput. Optim. Appl. 42(3): 371-392.

Da Silva, E. L., Gil, H. A. and Areiza, J. M. (2000). Transmission network expansion planning under an improved genetic algorithm, IEEE Trans. Power Syst. 15(4): 1168-1175.

Dietrich, B. L. and Escudero, L. F. (1990). Coefficient reduction for knapsack-like constraints in 0-1 programs with variable upper bounds, Operations Research Letters 9(1): 9-14.

Escobar, A. H., Gallego, R. A. and Romero, R. (2004). Multistage and coordinated planning of the expansion of transmission systems, IEEE Trans. Power Syst. 19(2): 735-744.

Garcia, B.-L., Mahey, P. and LeBlanc, L. J. (1997). Iterative improvement methods for a multiperiod network design problem, European Journal of Operational Research .

Garver, L. L. (1970). Transmission network estimation using linear programming, IEEE Trans. Power App. Syst. 89(7): 1688 - 1697.
Gauthier, J. M. and Ribiere, G. (1977). Experiments in mixed-integer linear programming using pseudo-costs, Mathematical Programming 12(1): 26-47.

Haffner, S., Monticelli, A., Garcia, A., Mantovani, J. and Romero, R. (2000). Branch and bound algorithm for transmission system expansion planning using a transportation model, IEEE Proceedings-Generation, Transmission and Distribution 147(3): 149-156.

Haffner, S., Monticelli, A., Garcia, A. and Romero, R. (2001). Specialised branch-and-bound algorithm for transmission network expansion planning, IEEE Proceedings-Generation, Transmission and Distribution 148(5): 482-488.

Land, A. and Doig, A. (1960). An automatic method of solving discrete programming problems, Econometrica: Journal of the Econometric Society pp. 497-520.

Levi, V. and Calovic, M. (1991). A new decomposition based method for optimal expansion planning of large transmission networks, IEEE Trans. Power Syst. 6(3): 937943.

Linderoth, J. and Savelsbergh, M. (1999). A computational study of search strategies for mixed integer programming, INFORMS Journal on Computing 11: 173-187.

Monticelli, A., Santos, A., Pereira, M., Cunha, S., Parker, B. and Praca, J. (1982). Interactive transmission network planning using a least-effort criterion, IEEE Trans. Power App. Syst. PAS-101: 3919-3925.

Murtagh, B. and Saunders, M. (1987). MINOS 5.4 User's Guide (preliminary), Department of Operations Research Stanford University, Technical report, Revised Jan.

Nemhauser, G. and Wolsey, L. (1999). Integer and combinatorial optimization, Wiley New York.

Padberg, M. and Rinaldi, G. (1987). Optimization of a 532city symmetric traveling salesman problem by branch and cut, Operations Research Letters 6(1): 1-7.

Padberg, M. and Rinaldi, G. (1991). A branch-and-cut algorithm for the resolution of large-scale symmetric traveling salesman problems, SIAM review 33(1): 60-100.

Pereira, M. and Pinto, L. (1985). Application of sensitivity analysis of load supplying capability to interactive transmission expansion planning, IEEE Trans. Power App. Syst. PAS-104: 381-389.

Romero, R., Gallego, R. A. and Monticelli, A. (1996). Transmission system expansion planning by simulated annealing, IEEE Trans. Power Syst. 11(1): 364-369. 
Romero, R., Mantovani, M., Rocha, C. and Mantovani, J. R. S. (2007). Evaluation of hybrid models for static and multistage transmission system planning, Sba: Controle \& Automação Sociedade Brasileira de Automatica 18: 106-114.

Romero, R. and Monticelli, A. (1994). A hierarchical decomposition approach for transmission network expansion planning, IEEE Trans. Power Syst. 9(3): 1385-1391.

Romero, R., Monticelli, A., Garcia, A. and Haffner, S. (2002). Test systems and mathematical models for transmission network expansion planning, IEEE Proceedings-Generation, Transmission and Distribution 149(1): 27-36.

Romero, R., Rocha, C., Mantovani, M. and Mantovani, J. (2003). Analysis of heuristic algorithms for the transportation model in static and multistage planning in network expansion systems, IEEE Proceedings-Generation, Transmission and Distribution 150(5): 521-526.

Sousa, A. S. and Asada, E. N. (2008). Tomada de decisão fuzzy aplicada ao planejamento da expansão de sistemas de transmissão, XVII Congresso Brasileiro de Automática, Juiz de Fora - MG.

Villasana, R., Garver., L. L. and Salon, S. J. (1985). Transmission Network Planning Using Linear Programming, IEEE Trans. Power App. Syst. PAS-104: 349-356.

Wolsey, L. (1998). Integer programming, Wiley New York. 